\title{
Evaporação líquida no lago de Sobradinho e impactos no escoamento devido à construção do reservatório ${ }^{1}$
}

\author{
$\overline{\text { Silvio B. Pereira² }}$, Fernando F. Pruski ${ }^{3}$, Demetrius D. da Silva ${ }^{3}$ \& Márcio M. Ramos ${ }^{3}$
}

\begin{abstract}
RESUMO
Um dos problemas que dificultam a gestão de recursos hídricos, são as perdas de água por evaporação nos reservatórios de regularização, fazendo com que estimativas confiáveis de evaporação nos reservatórios sejam essenciais, tanto no planejamento e gerenciamento dos recursos hídricos, quanto em estudos de impacto ambiental. Com base neste enfoque objetivou-se, através do trabalho ora apresentado, estimar a evaporação líquida no lago de Sobradinho por meio da metodologia do tanque Classe A e dos modelos Linacre (1993), Kohler et al. (1955) e CRLE, além dos impactos ocorridos nas condições de escoamento devido à construção do reservatório. Os resultados permitiram concluir que: a construção do reservatório de sobradinho alterou sensivelmente o comportamento hidrológico do rio São Francisco à jusante da mesma; os valores obtidos pelo modelo Kohler et al. (1955) podem ser utilizados como base de referência para a estimativa da evaporação média anual do lago de Sobradinho; o coeficiente de tanque Classe A que melhor representou a variação sazonal de evaporação no lago de Sobradinho foi de 0,57 ; finalmente, a vazão média anual correspondente à evaporação líquida do reservatório de Sobradinho foi de $132 \mathrm{~m}^{3} \mathrm{~s}^{-1}$.
\end{abstract}

Palavras-chave: aproveitamento hidrelétrico, evaporação em lagos, recursos hídricos

\section{Net evaporation in Sobradinho dam and impacts on runoff due to the construction of the reservoir}

\begin{abstract}
Losses of water by evaporation in regulatory reservoirs are one of the problems that exist in water resources management, the reliable estimates of evaporation in the reservoirs becoming essential not only in the planning and administration of the water resources but also in studies of environmental impact. The objective of this paper was to estimate the net evaporation in the Sobradinho dam through the Class A tank methodology and the Linacre (1993), Kohler et al. (1955) and CRLE models, as well as to verify the impacts on the flow conditions due to the construction of the reservoir. The results showed that: the construction of the Sobradinho reservoir, associated to the other activities developed in the basin, caused a reduction of the average flow of $163 \mathrm{~m}^{3} \mathrm{~s}^{-1}$; the values obtained by the Kohler et al. (1955) model can be used as a reference base to estimate the annual average evaporation in the Sobradinho dam; the coefficient of Class A tank that represented better the seasonal variation of evaporation in the Sobradinho dam was 0.57 and the annual average flow corresponding to net evaporation of Sobradinho reservoir was $132 \mathrm{~m}^{3} \mathrm{~s}^{-1}$.
\end{abstract}

Key words: hydroelectric power, regulated flow, water resources

\footnotetext{
1 Parte da tese de doutorado do primeiro autor apresentada à UFV

2 Faculdade de Ciências Agrárias/UFGD, CEP 79804-970, Cx. Postal 533, Dourados, MS. Fone: (67) 3411-3854. E-mail: sbueno@ufgd.edu.br

3 DEA/UFV, CEP 36571-000, Fone: (31) 3899-1912. E-mail: ffpruski@ufv.br, david@ufv.br, mmramos@ufv.br
} 


\section{INTRODUÇÃO}

Em 1973, a Companhia Hidro Elétrica do São Francisco (CHESF) começou a construção da hidrelétrica de Sobradinho, cujo lago, formado em 1979, com superfície de $4.214 \mathrm{~km}^{2}$, um dos maiores do mundo, serve como reservatório de regularização plurianual de vazão do Rio São Francisco. 0 reservatório de Sobradinho se localiza no Estado da $B$ ahia, acerca de $40 \mathrm{~km}$ à montante das cidades de J uazeiro, $B A$, e Petrolina, PE, cuja potência instalada é de 1.140 M W (Correia \& Dias, 2003).

Um fator de grande importância na análise da vazão máxima permissível para a concessão de outorga se refere às perdas de água por evaporação nos reservatórios de regularização. A obtenção dos valores de evaporação permite avaliar a quantidade de água que se perde ou se ganha com a construção de um reservatório ( $K$ an \& Dias, 1999); portanto, estimativas confiáveis de evaporação nos reservatórios são essenciais, tanto no planejamento e gerenciamento dos recursos hídricos como em estudos de impacto ambiental.

0 desenvolvimento de métodos confiáveis para estimativa da evaporação em lagos baseados em informações climatológicas de fácil obtenção, ainda é um desafio. A maioria dos métodos atualmente disponíveis estima a evaporação a partir de observações de temperatura, umidade, velocidade do vento e radiação solar ou usa as medidas de evaporação feitas em tanques instalados em estações meteorológicas situadas em ambiente terrestre (Roque \& Sansigolo, 2001).

Normalmente, os model os disponíveis para estimar a evaporação em lagos são divididos, em termos práticos, em duas classes: nos em que se utilizam equações baseadas em informações climatológicas para a predição da evaporação, como os modelos propostos por M orton (1983), Linacre (1993) e Kohler et al. (1955), entre outros, naqueles em que se empregam medidas de tanques de evaporação.

Um dos principais problemas na utilização de modelos climatológicos na estimativa da evaporação é a escassez de dados climatológicos sobre a superfície do lago, fazendose uso das informações climatológicas de estações situadas em ambiente terrestre e, portanto, não representativas do ambiente sobre o lago; no caso do uso de medidas realizadas em tanques, é a escol ha apropriada do coeficiente do tanque (Reis \& Dias, 1998).

Com base neste enfoque é que se teve, neste trabal ho, como objetivo principal, estimar a evaporação líquida no lago de Sobradinho com o emprego da metodologia do tanque Classe A e dos model os L inacre (1993), K ohler et al. (1955) e CRLE, além dos impactos ocorridos nas condições de escoamento em virtude da construção do reservatório de Sobradinho.

\section{MATERIAL E MÉTODOS}

Com o objetivo de avaliar os impactos ocorridos nas condições de escoamento do Rio São Francisco, devido à construção do reservatório de Sobradinho, utilizou-se a série original da estação J uazeiro, localizada à jusante do reservatório de Sobradinho, e a obtida a partir da extensão de série, e também as informações das estações Ibotirama (área de drenagem de $325.200 \mathrm{~km}^{2}$ ) e M orpará (área de drenagem de $348.074 \mathrm{~km}^{2}$ ) situadas à montante de J uazeiro e o período de dados comum anterior à construção do reservatório de Sobradinho; portanto, a extensão de série para J uazeiro a partir dos dados das estações de apoio, permitiu que se projetasse o comportamento esperado em Juazeiro caso não ocorresse a interferência advinda da criação do lago.

Realizou-se a extensão de série para J uazeiro por meio de correlações, segundo critérios consagrados de escolha de bases para as regressões, sendo os coeficientes de determinação obtidos para o período de 1950 a 1976 de 0,91 entre J uazeiro e Ibotirama e de 0,84 , entre Juazeiro e M orpará.

A estimativa da evaporação no lago de Sobradinho foi calculada pelas seguintes metodologias: tanque Classe A (ECA), modelo de Linacre (1993), modelo de Kohler et al. (1955) e modelo conceitual de relação complementar (CRLE), proposto por Morton (1983).

Fez-se a estimativa da evaporação no lago pelo método do tanque Classe $A$, pela equação:

$$
\mathrm{E}_{\mathrm{L}}=\mathrm{C} \mathrm{ECA}
$$

em que:

$E_{L}$ - evaporação no lago, mm mês-1

$C$ - coeficiente do tanque, adimensional

ECA - evaporação no tanque Classe $A, \mathrm{~mm}_{\text {mês }}{ }^{-1}$

Os coeficientes de tanque normalmente utilizados para a estimativa da evaporação em lagos, são de 0,6 a 0,8. Neste trabal ho se adotou o coeficiente de 0,6 , em função de ser este o valor de uso mais comum em regiões áridas, como é o caso do reservatório de Sobradinho.

A equação utilizada para a estimativa da evaporação no lago de Sobradinho por meio do modelo proposto por Linacre (1993) corresponde a uma simplificação da equação de Penman quando os dados de temperatura média, precipitação e velocidade do vento são conhecidos. A equação seguinte foi utilizada:

$E_{L}=\left(0,015+0,00042 T+10^{-6} h\right)\left[0,8 R_{s}-40+2,5 \mathrm{Fu}_{2}\left(T-T_{d}\right)\right]$

em que:

$\mathrm{T}$ - temperatura média do $\mathrm{ar},{ }^{\circ} \mathrm{C}$

$T_{d}$ - temperatura média do ponto de orvalho, ${ }^{\circ} \mathrm{C}$

$\mathrm{h}$ - altitude do logal, $\mathrm{m}$

$R_{S}$ - irradiância solar na superfície do lago, $\mathrm{W} \mathrm{m}^{-2}$

F - fator de correção, adimensional

$\mathrm{u}_{2}$ - velocidade do vento a $2 \mathrm{~m}$ de altura, $\mathrm{m} \mathrm{s}^{-1}$

0 modelo proposto por K ohler et al. (1955) também consiste em uma adaptação da equação de Penman, feita a partir de várias observações de evaporações em lagos. Para a estimativa da evaporação em lagos pelo modelo Kohler et al. (1955) empregou-se a equação:

$$
\mathrm{E}_{\mathrm{L}}=0,7\left(\frac{\Delta \mathrm{R}_{\mathrm{n}}}{\Delta+\gamma_{\mathrm{L}}}+\frac{\gamma_{\mathrm{L}} \mathrm{E}_{\mathrm{a}}}{\Delta+\gamma_{\mathrm{L}}}\right)
$$

em que: 
$\Delta$ - declividade da curva de pressão de vapor saturado, $\mathrm{kPa}^{\circ} \mathrm{C}^{-1}$

$R_{n}$ - saldo de radiação, em equivalente de lâmina de água evaporada, $\mathrm{mm} \mathrm{dia}^{-1}$

$\gamma_{\mathrm{L}}$ - coeficiente psicrométrico corrigido, $\mathrm{kPa}{ }^{\circ} \mathrm{C}^{-1}$

$E_{a}$ - poder evaporante do ar, mm dia-1

A equação utilizada para estimar a evaporação no lago de Sobradinho, por meio do modelo CRLE, foi obtida da modificação da equação de Piestley e Taylor (M orton, 1983) com vista a atender ao modelo CRLE, sendo e expressa por:

$$
\mathrm{E}_{\mathrm{W}}=13+1,12\left(1+\frac{0,66 \mathrm{P} / \mathrm{P}_{\mathrm{S}}}{\Delta_{\mathrm{P}}}\right)^{-1} \mathrm{R}_{\mathrm{TP}}
$$

em que:

$E_{W}$ - evaporação no lago, mm mês ${ }^{-1}$

$P$ - pressão atmosférica na localidade, mb

$P_{S}$ - pressão atmosférica a nível do mar, mb

$\Delta_{\mathrm{P}}$ - declividade da curva de pressão de saturação do vapor d'água a temperatura de equilíbrio, $m b{ }^{\circ} \mathrm{C}^{-1}$

$R_{T P}$ - saldo de radiação a temperatura de equilíbrio, $\mathrm{W} \mathrm{m}^{-2}$

Os valores 13 e 1,12, apresentados na Eq. 6, são constantes empíricas obtidas por meio de calibrações em diversas regiões áridas do mundo. Os cálculos para a estimativa da evaporação obtida pelo modelo CRLE foram realizados segundo a metodologia proposta por M orton (1983).

A estimativa da evapotranspiração real concernente ao período anterior à construção do reservatório de Sobradinho, foi realizada com o método do balanço hídrico climatológico proposto por Thorntwaite $\&$ M ather (1955). Partindo do suprimento natural de água ao solo, representado pela precipitação e da demanda atmosférica, representada pela evapotranspiração potencial, o balanço hídrico climatológico fornece estimativas da evapotranspiração real, da deficiência, do excedente e do armazenamento de água no solo.

A estimativa da evapotranspiração potencial, parâmetro de entrada no modelo, foi baseada na metodologia proposta por Penman-M onteith, contida na publicação da FAO 56 (Allen et al., 1998).

Obtiveram-se os valores de evaporações líquidas médias mensais para o reservatório de Sobradinho pela diferença entre a evaporação do lago, estimada por meio de modelos climatológicos, e a evapotranspiração real na área correspondente ao reservatório de Sobradinho, antes da sua construção.

Os dados climatológicos utilizados neste trabal ho, foram: temperatura média mensal, umidade relativa, velocidade do vento, precipitação, evaporação no tanque Classe $\mathrm{A}$ e insolação. Na Tabela 1 se apresentam as estações utilizadas, coordenadas geográficas e períodos considerados na análise. Para o período anterior à construção do reservatório de Sobradinho, utilizaram-se as informações climatológicas fornecidas pela Agência Nacional das Águas (ANA) e, para 0 período posterior às informações, foram fornecidas pela Companhia Hidroelétrica do São Francisco (CHESF).

Realizou-se a análise de sensibilidade para os modelos
Tabela 1. Estações climatológicas utilizadas no estudo

\begin{tabular}{|c|c|c|c|c|}
\hline Estação & Latitude & Longitude & Altitude (m) & Período (19...) \\
\hline Cabrobó & $8^{\circ} 18^{\prime} 36^{\prime \prime}$ & $39^{\circ} 12^{\prime} 00^{\prime \prime}$ & 341 & $\begin{array}{l}63-70 \\
73-77\end{array}$ \\
\hline Floresta & $8^{\circ} 21^{\prime} 36^{\prime \prime}$ & $38^{\circ} 20^{\prime} 24^{\prime \prime}$ & 309 & $\begin{array}{l}61-70 \\
63-77\end{array}$ \\
\hline Petrolina & $9^{\circ} 13^{\prime} 48^{\prime \prime}$ & $40^{\circ} 18^{\prime} 00^{\prime \prime}$ & 370 & $\begin{array}{l}63-64 \\
73-77\end{array}$ \\
\hline Petrolândia & $9^{\circ} 02^{\prime} 24^{\prime \prime}$ & $38^{\circ} 11^{\prime} 24^{\prime \prime}$ & 286 & $\begin{array}{c}61-70 \\
74 \\
80,84-89\end{array}$ \\
\hline Sobradinho & $9^{\circ} 25^{\prime} 00^{\prime \prime}$ & $40^{\circ} 49^{\prime} 60^{\prime \prime}$ & 371 & $\begin{array}{l}91,93,94 \\
96,97,99\end{array}$ \\
\hline
\end{tabular}

Linacre (1993), K ohler et al. (1955) e CRLE, conduzida para os parâmetros de entrada dos modelos, tais como: precipitação, temperatura média, velocidade do vento e insolação, variando-os, individualmente, enquanto os outros eram mantidos constantes. A análise de sensibilidade quantifica o efeito que as mudanças nas variáveis de entrada exercem na evaporação média anual, no reservatório de Sobradinho.

\section{RESULTADOSE DISCUSSÃO}

A presentam-se, na Tabela 2, para a estação J uazeiro, as vazões médias anual e mensais referentes aos períodos de 1950 a 1976 e de 1979 a 2000 compreendendo, portanto, os períodos anterior e posterior à construção do reservatório, respectivamente.

Pode-se, na Tabela 2, não só contemplar a análise dos dados originais mas, também, considerar as séries pertinentes aos dados preenchidos a partir das estações Ibotirama e M orpará, as quais também estão localizadas no rio São F rancisco à montante do reservatório de Sobradinho não apresentando, portanto, a influência do reservatório de acumulação no comportamento do curso d'água.

Conforme se evidencia na Tabela 2, foi transparente a diferença entre as vazões médias anuais obtidas para J uazeiro (período de 1979 a 2000) a partir da série original, cujo valor é de $2.557 \mathrm{~m}^{3} \mathrm{~s}^{-1}$, e a estimada quando do preenchimento, igual a $2.720 \mathrm{~m}^{3} \mathrm{~s}^{-1}, 0$ que corresponde a uma diferença de $162 \mathrm{~m}^{3} \mathrm{~s}^{-1}(6,3 \%)$ por ocasião do preenchimento, a partir de Ibotirama, e a $2.722 \mathrm{~m}^{3} \mathrm{~s}^{-1}$, quando do preenchimento a partir de Morpará, correspondendo a uma diferença de $164 \mathrm{~m}^{3} \mathrm{~s}^{-1}(6,4 \%)$; este fato indica a alteração do comportamento do escoamento no rio São Francisco à jusante de Juazeiro a partir de 1979, com redução da vazão média observada em relação à estimada, da ordem de $163 \mathrm{~m}^{3} \mathrm{~s}^{-1}$.

Nota-se que o comportamento evidenciado pela análise feita a partir de Ibotirama, é bastante similar ao obtido a partir de Morpará, o que constitui forte indicativo da consistência da análise realizada.

Constata-se, ainda, que a vazão média observada para a estação M orpará (área de drenagem de 348.074 km²) igual a $2.710 \mathrm{~m}^{3} \mathrm{~s}^{-1}$ é, inclusive, superior à evidenciada em J uazeiro (área de drenagem de $510.800 \mathrm{~km}^{2}$ ), igual a 
Tabela 2. Vazões médias anual e mensais $\left(\mathrm{m}^{3} \mathrm{~s}^{-1}\right)$ na estação Juazeiro para os períodos de 1950 a 1976 e de 1979 a 2000

\begin{tabular}{|c|c|c|c|c|c|c|c|c|}
\hline \multirow{3}{*}{$\begin{array}{c}\text { Tempo } \\
\text { Anual/Mensal }\end{array}$} & \multirow{2}{*}{\multicolumn{2}{|c|}{$\begin{array}{c}\text { Dados Originais } \\
\text { Período }\end{array}$}} & \multirow{3}{*}{$\begin{array}{c}\begin{array}{c}\text { Dados } \\
\text { Preenchidos } \\
\text { (lbotirama) }\end{array} \\
\text { Período } \\
1979 \text { a } 2000\end{array}$} & \multicolumn{2}{|c|}{$\Delta Q^{*}$} & \multirow{3}{*}{$\begin{array}{c}\begin{array}{c}\text { Dados } \\
\text { Preenchidos } \\
\text { (Morpará) }\end{array} \\
\text { Período } \\
1979 \text { a } 2000\end{array}$} & \multicolumn{2}{|c|}{$\Delta Q^{*}$} \\
\hline & & & & \multirow{2}{*}{ Valor Absoluto } & \multirow{2}{*}{$(\%)$} & & \multirow{2}{*}{ Valor Absoluto } & \multirow{2}{*}{$(\%)$} \\
\hline & 1950 a 1976 & 1979 a 2000 & & & & & & \\
\hline Anual & 2.461 & 2.557 & 2.720 & 162 & 6,3 & 2.722 & 164 & 6,4 \\
\hline Janeiro & 3.938 & 3.162 & 4.282 & 1.120 & 35,4 & 4.291 & 1.128 & 35,7 \\
\hline Fevereiro & 4.239 & 3.618 & 5.128 & 1.510 & 41,7 & 5.125 & 1.507 & 41,6 \\
\hline Março & 3.948 & 3.768 & 4.515 & 746 & 19,8 & 4.669 & 900 & 23,9 \\
\hline Abril & 3.592 & 3.193 & 3.628 & 435 & 13,6 & 3.665 & 472 & 14,8 \\
\hline Maio & 2.191 & 2.336 & 2.507 & 172 & 7,4 & 2.442 & 107 & 4,6 \\
\hline Junho & 1.594 & 2.009 & 1.780 & -229 & $-11,4$ & 1.752 & -256 & $-12,8$ \\
\hline Julho & 1.393 & 1.950 & 1.549 & -400 & $-20,5$ & 1.535 & -415 & $-21,3$ \\
\hline Agosto & 1.248 & 1.985 & 1.420 & -565 & $-28,5$ & 1.404 & -582 & $-29,3$ \\
\hline Setembro & 1.118 & 2.029 & 1.308 & -721 & $-35,6$ & 1.306 & -723 & $-35,6$ \\
\hline Outubro & 1.242 & 2.043 & 1.385 & -658 & $-32,2$ & 1.382 & -661 & $-32,4$ \\
\hline Novembro & 1.882 & 2.146 & 1.993 & -153 & $-7,1$ & 1.969 & -177 & $-8,3$ \\
\hline Dezembro & 3.247 & 2.512 & 3.282 & 770 & 30,7 & 3.263 & 750 & 29,9 \\
\hline
\end{tabular}

$\Delta Q$ - Diferença entre a vazão preenchida e a vazão original

$2.557 \mathrm{~m}^{3} \mathrm{~s}^{-1}$, comprovando o impacto que a construção do reservatório e outras atividades desenvolvidas nesse trecho da bacia, teve no comportamento das vazões verificadas no rio São Francisco uma vez que, mesmo com o aumento da área de drenagem de 162.726 km² $^{2}$ (acréscimo de $68,1 \%$ na área de drenagem) se evidencia um declínio da vazão média anual, de $153 \mathrm{~m}^{3} \mathrm{~s}^{-1}$.

A análise da variação das vazões médias mensais permite evidenciar um nítido efeito de regularização exercido pelo reservatório sendo que, de dezembro a maio, as vazões obtidas com o preenchimento foram superiores aos valores originais; nos meses de dezembro a março, a diferença entre as vazões originais e aquelas estimadas, considerando-se a tendência do comportamento das vazões observadas anteriores a 1976, tanto para Ibotirama como para M orpará, é de mais de $740 \mathrm{~m}^{3} \mathrm{~s}^{-1}$, fato que mostra o efeito exercido pelo reservatório no controle de enchentes o qual ocasionou uma redução das vazões máximas à jusante do reservatório.

Para o período de estiagem, as vazões médias mensais estimadas a partir do preenchimento são inferiores àquelas verificadas no rio, tendo-se observado, nos meses de junho a novembro, vazões estimadas a partir da extensão da série menores que as notadas; essas diferenças chegaram a atingir valores de até $723 \mathrm{~m}^{3} \mathrm{~s}^{-1}$, sendo a menor média mensal da série original igual a $1.950 \mathrm{~m}^{3} \mathrm{~s}^{-1}$, constatada em julho, enquanto 0 menor valor relativo às séries com valores preenchidos é de $1.306 \mathrm{~m}^{3} \mathrm{~s}^{-1}$ (considerando-se o preenchimento a partir de Morpará), evidenciada em setembro, o que mostra, inclusive, a mudança do mês com ocorrência da menor vazão à jusante de Juazeiro, que passou de setembro (anterior a 1976) para julho (posterior a 1979).

Embora a construção do reservatório de Sobradinho, associada às demais atividades desenvolvidas na bacia, tenha causado redução da vazão média da ordem de $163 \mathrm{~m}^{3} \mathrm{~s}^{-1}$, promoveu também um expressivo efeito de regularização com elevação da vazão média mensal correspondente ao mês mais seco, de $1.306 \mathrm{~m}^{3} \mathrm{~s}^{-1}$ para $1.950 \mathrm{~m}^{3} \mathrm{~s}^{-1}$ (aumento de $\left.644 \mathrm{~m}^{3} \mathrm{~s}^{-1}\right)$, tendo em vista as vazões projetadas a partir de M orpará, e decréscimo da vazão mensal relativa ao mês com maior vazão de $5.128 \mathrm{~m}^{3} \mathrm{~s}^{-1}$ (considerando-se vazões projetadas a partir de Ibotirama) para $3.768 \mathrm{~m}^{3} \mathrm{~s}^{-1}$ (diminuição de $1.360 \mathrm{~m}^{3} \mathrm{~s}^{-1}$ ).

\section{Evaporação no lago de Sobradinho}

A Figura 1 apresenta as evaporações médias mensais estimadas pelo método do tanque Classe $A$ e pelos modelos Linacre (1993), Kohler et al. (1955) e CRLE. A s evaporações totais anuais estimadas por esses modelos, foram: $2.149 \mathrm{~mm}$ (Linacre, 1993), $2.026 \mathrm{~mm}$ (ECA), $1.904 \mathrm{~mm}$ (Kohler et al., 1955) e $1.796 \mathrm{~mm}$ (CRLE).

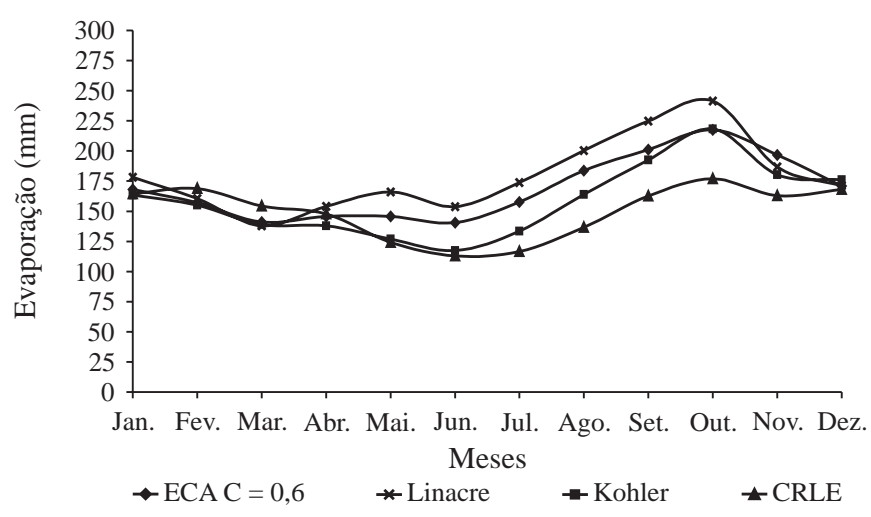

Figura 1. Evaporação no lago de Sobradinho, estimada pelo método do tanque Classe A e pelos modelos Linacre (1993), Kohler et al. (1955) e CRLE, considerando-se o período de 1980 a 1999

Tendo em vista que o método do tanque Classe A apresentou valor médio entre os modelos Linacre (1993) e K ohler et al. (1955) e respondeu diretamente às condições climáticas da região, ele foi adotado como modelo-padrão na comparação dos comportamentos evidenciados pelos demais modelos. 
Evidenciam-se dois efeitos distintos pela análise da Figura 1, os quais compreendem, de modo geral, expressivo aumento da evaporação a partir de junho (em outubro, todos os modelos atingem o valor máximo) e redução a partir de outubro, prolongada até junho, mês em que os valores foram mínimos em todos os modelos, com exceção de Linacre (1993), que apresentou a menor estimativa da evaporação em março.

A estimativa da evaporação obtida pelos model os estudados pode ser melhor entendida pela análise de sensibilidade a variação da temperatura, velocidade do vento, precipitação e insolação, como mostrado na Figura 2.

Considerando-se o mês de maior evaporação no reservatório de Sobradinho (outubro), que se caracteriza pela baixa precipitação e umidade relativa, pela máxima insolação e por valores mais elevados de temperatura e velocidade de vento, observa-se que o modelo Linacre (1993) é o que apresenta a maior estimativa da evaporação no reservatório.

Pela análise de sensibilidade apresentada na Figura 2, 0 modelo Linacre (1993) é o único sensível à variação da precipitação, conforme pode ser evidenciado pela declividade da linha que representa este parâmetro (Figura $2 \mathrm{~A}$ ); sendo baixa a precipitação em outubro, o modelo L inacre (1993) tende a uma estimativa maior da evaporação em relação aos modelos Kohler et al. (1955) e CRLE.

Outro fator que contribui para a maior estimativa da evaporação pelo uso do modelo Linacre (1993), é a sensibilidade que este apresenta à variação da velocidade do vento, principal mente quando comparado com o modelo CRLE, que não indica sensibilidade a esta variável climática. A alta velocidade de vento em outubro ocasionou maior aumento na estimativa de evaporação pelo modelo Linacre (1993) porém, com relação ao modelo Kohler et al. (1955), notouse que as declividades das linhas representadas pelas variações desse parâmetro (Figura 2B) são praticamente iguais, não sendo, portanto, o fator que conduziu a diferença na estimativa da evaporação nesse mês.

E $m$ relação à análise de sensibilidade a temperatura (Figura 2C), o modelo Linacre (1993) é menos sensível em comparação com os modelos Kohler et al. (1955) e CRLE, fazendo com que 0 aumento da temperatura em outubro ocasionasse maior aproximação entre eles, bem como a falta de sensibilidade a insolação, pelo modelo Linacre (1993) e as altas sensibilidades dos modelos K ohler et al. (1955) e CRLE (Figura 2D).

Constatou-se, também, que o modelo K ohler et al. (1955) apresentou um valor de evaporação muito acima do estimado por CRLE, fato que pode ser explicado pela análise da velocidade do vento e pela temperatura, as quais o modelo Kohler et al. (1955) apresentou maior e menor sensibilidade, respectivamente.

0 mês de menor evaporação no reservatório de Sobradinho (junho) correspondeu ao final do período chuvoso, com exceção do modelo L inacre (1993); este mês se caracterizava por baixas precipitação, temperatura e insolação e alta umidade relativa e velocidade de vento correspondente à média anual. A maior proximidade na estimativa da evaporação entre os modelos Kohler et al. (1955) e CRLE pode ser explicada por: maior sensibilidade dos modelos $\mathrm{K}$ ohler
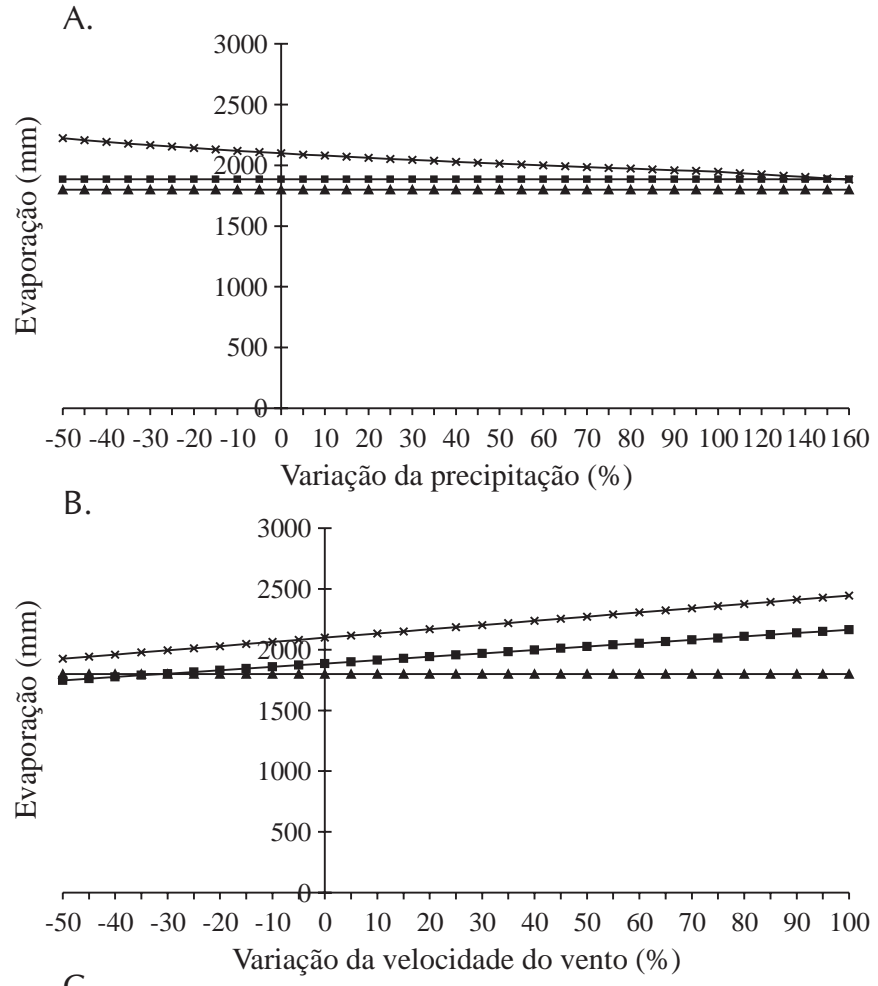

C.

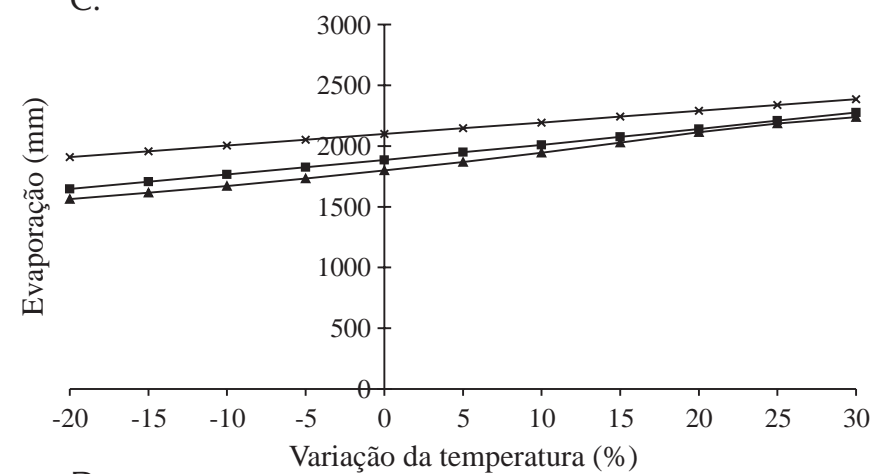

D.

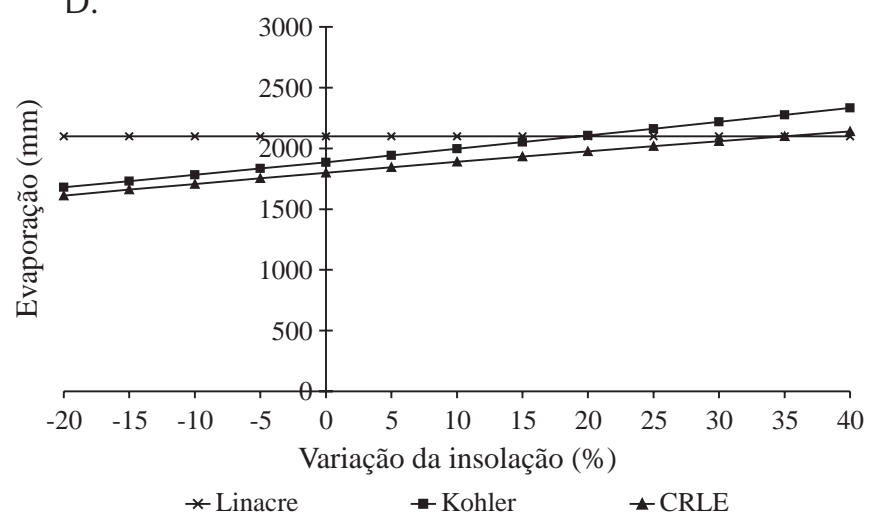

Figura 2. Sensibilidade dos modelos Linacre (1993), Kohler et al. (1955) e CRLE aos parâmetros climáticos relativos à precipitação $(A)$, velocidade do vento (B), temperatura (C) e insolação (D)

et al. (1955) e CRLE a temperatura, tendo-se identificado, no mês, baixa temperatura, e ocorrência de velocidade de vento, ainda não expressiva na região. 0 aumento das diferenças entre os modelos Kohler et al. (1955) e CRLE em relação ao Linacre (1993) (Figura 2) ocorreu principalmente devido às baixas precipitação e insolação. 
A alta precipitação evidenciada em março (157 mm), correspondendo a cerca de $30 \%$ do total anual precipitado e a baixa velocidade do vento $\left(1,1 \mathrm{~m} \mathrm{~s}^{-1}\right)$ fizeram com que 0 modelo Linacre (1993) apresentasse a menor estimativa da evaporação no mês; outro fato considerado em março foi a maior estimativa da evaporação obtida pelo modelo CRLE, que estava relacionada com a maior sensibilidade do modelo a temperatura e com a falta de sensibilidade à velocidade do vento.

Observou-se que, de modo geral, o model o Linacre (1993) tendeu a uma estimativa maior da evaporação em toda a faixa de variação contemplada na análise de sensibilidade; entretanto, quando a precipitação, a temperatura e a insolação aumentavam e a velocidade do vento diminuía, os modelos estudados tendiam a convergir para uma mesma estimativa da evaporação fato evidenciado principalmente nos meses de janeiro e dezembro.

\section{Evaporação líquida no lago de Sobradinho}

A presentam-se, na Tabela 3, a estimativa da evapotranspiração real obtida pelo balanço hídrico climático, segundo o método proposto por Thornthwaite \& Mather (1955), a evapotranspiração potencial e a precipitação na região estudada antes da implantação do reservatório de Sobradinho.

Verifica-se, através da análise da Tabela 3, uma evapotranspiração potencial superior à precipitação total anual fazendo com que ocorra déficit hídrico muito acentuado na localidade do reservatório de Sobradinho e que a evapotranspiração real fique limitada ao total precipitado, uma vez que a evapotranspiração real não poderia superar a precipitação evidenciada na região.

$\mathrm{Na}$ Figura 3 se apresenta a evaporação líquida no lago de Sobradinho estimada pelos modelos do tanque Classe A, Linacre (1993), Kohler et al. (1955) e CRLE, a qual foi calculada subtraindo-se as evaporações do lago estimadas pelos modelos da evapotranspiração real. Os comportamentos foram semelhantes aos da evaporação do lago; entretanto, a queda nos valores calculados foi mais acentuada em março, em decorrência da alta evapotranspiração real ocasionada pela el evação da precipitação média mensal.

A s evaporações líquidas totais anuais estimadas pelos modelos tanque Classe A, Linacre (1993), Kohler et al. (1955) e CRLE, foram de 1.452, 1.575, 1.330 e 1.222 mm, respectivamente.

Com os valores das estimativas das evaporações líquidas e conhecidas as áreas de acumulação do lago de Sobradinho (fornecida, pela CHESF), calcularam-se as vazões decorrentes dessas evaporações, apresentadas na Tabela 4. A comparação entre os quatros modelos utilizados permitiu evidenciar que o modelo CRLE, embora seja o de mai-

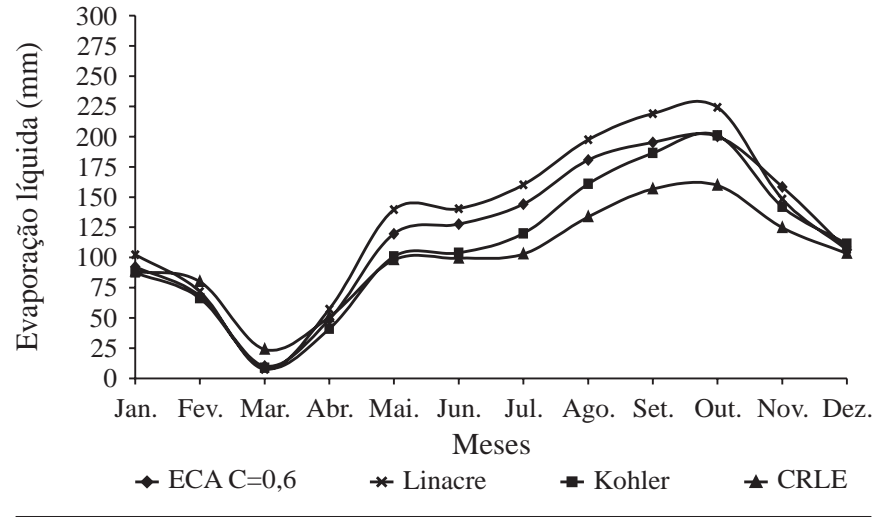

Figura 3. Evaporação líquida $(\mathrm{mm})$ no lago de Sobradinho estimada pelos modelos do tanque Classe A, Linacre (1993), Kohler et al. (1955) e CRLE, considerando-se o período de 1980 a 1999

or complexidade de utilização, foi o que apresentou o comportamento com maior discrepância em relação aos demais subestimando, inclusive, a evaporação no reservatório, fato que ocorreu, basicamente, em virtude da falta de sensibilidade do modelo à velocidade do vento.

Tabela 4. Vazão líquida evaporada no reservatório de Sobradinho, estimada com o uso dos modelos do tanque Classe A (ECA C $=0,6)$, Linacre (1993), Kohler et al. (1955) e CRLE

\begin{tabular}{lc}
\hline Modelos & Vazão líquida evaporada $\left(\mathbf{m}^{3} \mathbf{s}^{-1}\right)$ \\
ECA C $=0,6$ & 140 \\
Linacre (1993) & 155 \\
Kohler et al. (1955) & 130 \\
CRLE & 120 \\
\hline
\end{tabular}

Tendo em vista que o modelo Kohler et al. (1955) apresenta uma base conceitual mais fundamentada na equação original de Penman e se levando em conta, ainda os termos aerodinâmicos e 0 saldo de radiação, considera-se que os valores estimados pelo modelo Kohler et al. (1955) podem ser utilizados como boa alternativa na determinação da evaporação média anual no lago de Sobradinho. 0 modelo Linacre (1993), apesar de baseado na equação original de Penman considera, na estimativa do saldo de radiação, os dados de chuva, diferentemente do modelo K ohler et al. (1955), que se baseia apenas na insolação.

Considerando-se que o modelo do tanque Classe A representa bem a variação sazonal da evaporação e que o principal problema desse método reside na determinação do coeficiente do tanque sugere-se, como procedimento alternativo para representação da evaporação do reservatório de Sobradinho, a correção dos valores obtidos pelo método do tanque Classe A

Tabela 3. Evapotranspiração real (ETR, mm), evapotranspiração potencial (ETP, mm) e precipitação (Ppt, mm) na região do reservatório de Sobradinho antes da sua formação, segundo o método do balanço hídrico de Thornthwaite \& Mather (1955)

\begin{tabular}{|c|c|c|c|c|c|c|c|c|c|c|c|c|c|}
\hline Mês & Jan. & Fev. & Mar. & Abr. & Maio & Jun. & Jul. & Ago. & Set. & Out. & Nov. & Dez. & Total \\
\hline $\mathrm{E}_{\mathrm{TR}}$ & 75,9 & 88,7 & 130,2 & 96,9 & 26,2 & 13,4 & 13,6 & 2,9 & 6,0 & 17,4 & 38,3 & 64,7 & 574,2 \\
\hline $\mathrm{E}_{\mathrm{TP}}$ & 171,2 & 151,3 & 144,2 & 132,9 & 130,9 & 118,3 & 126,9 & 156,8 & 173,8 & 193,6 & 183,3 & 177,0 & 1860,2 \\
\hline $\mathrm{P}_{\mathrm{pt}}$ & 75,9 & 88,7 & 130,2 & 96,9 & 26,2 & 13,4 & 13,6 & 2,9 & 6,0 & 17,4 & 38,3 & 64,7 & 574,2 \\
\hline
\end{tabular}


a partir do uso de um coeficiente de tanque que represente a relação entre o valor de evaporação obtido pelo modelo de Kohler et al. (1955) e as evaporações medidas no tanque Classe A; esta correção conduz a um valor de 0,57 ao invés de 0,6 , inicialmente utilizado como coeficiente de tanque; portanto, levando-se em conta os valores obtidos por este procedimento, a vazão média anual correspondente à evaporação líquida no lago de Sobradinho foi de $132 \mathrm{~m}^{3} \mathrm{~s}^{-1}$ sendo, na análise mensal, a vazão líquida evaporada de maior magnitude de $211 \mathrm{~m}^{3} \mathrm{~s}^{-1}$, ocorrida em setembro.

Comparando-se o valor obtido pela redução da vazão média anual $\left(163 \mathrm{~m}^{3} \mathrm{~s}^{-1}\right)$, devido à construção do reservatório de Sobradinho, associada às demais atividades desenvolvidas na bacia, com a vazão média anual correspondente à evaporação líquida (132 $\left.\mathrm{m}^{3} \mathrm{~s}^{-1}\right)$, tem-se, por diferença, o valor da ordem de $31 \mathrm{~m}^{3} \mathrm{~s}^{-1}$, sendo este o valor médio associado às demais atividades desenvolvidas no trecho entre M orpará e Juazeiro. De acordo com ONS et al. (2003), em estudos realizados visando à estimativa das vazões para atividade de uso consuntivo da água nas principais bacias do sistema interligado nacional, se obteve, para a bacia do São Francisco, no mesmo trecho, o valor da ordem de $20 \mathrm{~m}^{3} \mathrm{~s}^{-1}$, considerando-se 0 ano de 1996, que compreende o último ano de censo agropecuário durante a realização do trabalho.

\section{CONCLUSÕES}

1. A construção do reservatório de Sobradinho, associada às demais atividades desenvolvidas na bacia, causou redução da vazão média, da ordem de $163 \mathrm{~m}^{3} \mathrm{~s}^{-1}$.

2. Os valores obtidos pelo modelo de Kohler et al. (1955) podem ser utilizados como base de referência para a estimativa da evaporação média anual do lago de Sobradinho.

3. O coeficiente de tanque Classe A que melhor representou a variação sazonal de evaporação no lago de Sobradinho, foi 0,57 .

4. A vazão média anual correspondente à evaporação líquida do reservatório de Sobradinho, foi de $132 \mathrm{~m}^{3} \mathrm{~s}^{-1}$.

\section{LITERATURA CITADA}

Allen, R. G.; Pereira, L. S.; Raes, D.; Smith, M. Grop evapotranspiration: Guidelines for computing crop water requirements. FA 0 irrigation and drainage. Roma: Food and A griculture Organization of the U nited Nations, 1998. 328p. Paper, 56

Correia, M. F.; Dias, M. A. F. S. Variação do nível do reservatório de Sobradinho e seu impacto sobre o clima da região. Revista Brasileira de Recursos Hídricos, v.8, n.1, p.157-168, 2003.

Kan, A.; Dias, N. L. Evaporação, evapotranspiração e evaporação líquida no reservatório de $\mathrm{F} 0 \mathrm{z}$ do A reia. Revista B rasileira de Recursos Hídricos, v.4, n.3, p.29-38, 1999.

K ohler, M. A.; N ordensen, T. J.; F ox, W. E. Evaporation from pans and lakes, Washington: U. S. Weather Bureau Research, 1955. 21p. Paper 38

Linacre, E. T. Data-sparse estimation of lake evaporation using a simplified Penman equation. Elsevier Science Publishers B. V. A gricultural and Forest M eteorology, v.64, p.237-256, 1993.

M orton, F. I. Operational estimates of lake evaporation. Journal of hydrology,A msterdam, v.66, n.3, p.77-100, 1983.

ONS; FAHMA; DREER. Estimativa das vazões para atividades de uso consuntivo da água nas principais bacias do sistema interligado nacional - M etodologia e resultados consolidados. Brasília: Operadora Nacional do Sistema Elétrico - Consórcio FAHMA/DREER, 2003. v.1. 209p.

Reis, R. J.; Dias, N. L. M ulti-season lake evaporation: energu-budget estimates and CRLE model assessment with limited meteorological observations. Journal of Hydrology, v.208, n.3-4, p.135-147, 1998.

Roque, R. 0.; Sansigolo, C. A. Estimativas de evaporação do lago Taquaruçu, SP pelo modelo conceitual de M orton. Revista Brasileira de Recursos Hídricos, v.6, n.1, p.21-28, 2001.

Thornthwaite, C. W.; M ather, J. R. The water balance. Publications in Climatology. New Jersey: Drexel Institute of Technology, 1955. 104p. 